\title{
HYSIM: Hybrid Fault Simulation for Synchronous Sequential Circuits
}

\author{
KYUCHULL KIM and KEWAL K. SALUJA* \\ Department of Electrical and Computer Engineering, University of Wisconsin-Madison, 1415 Johnson Drive, Madison, WI 53706-1691
}

\begin{abstract}
The paper identifies the inefficiencies of the critical processes in concurrent fault simulation and proposes methods to remove such inefficiencies in a systematic manner. Also, proposed are dynamic memory usage reduction strategies for concurrent fault simulators. Through extensive step-by-step experimentation, we verified the effectiveness of the proposed methods for performance improvement and identified best memory management strategy for dynamic memory usage reduction. A simulator, HySim, based on the proposed methods is implemented and shown to outperform the existing fault simulators and achieve dramatic memory usage reduction. The HySim maintains fault lists which are subsets of that of a conventional concurrent fault simulator, which yields shorter fault list processing time and reduced dynamic memory usage. It also employs Release-and-Reconstruct method for fault list construction, where any fault list identified to be useless is released immediately. The experimental results show that Release-and-Reconstruct method is very effective in dynamic memory usage reduction.
\end{abstract}

Keywords: Dynamic memory usage, Converged fault detection, Fault descriptor, Fault simulation, Hybrid fault simulation, Hysim, Synchronous sequential circuits

\section{INTRODUCTION}

Fault simulation algorithms for synchronous sequential circuits which have appeared in the literature can be categorized as follows: 1) serial fault simulation, 2) deductive fault simulation, 3 ) concurrent fault simulation. Serial fault simulator simulates a good machine and faulty machines one by one. Even though it is a straightforward way of simulation, it is impractical for simulating a large number of faults because it would consume an excessive amount of CPU time [1]. Deductive fault simulator [4] simulates a good circuit explicitly and determines detected faults by deduction. It processes all faults at the same time. Although deductive fault simulation is capable of 2-value and 3-value fault simulation, its use for 3-value simulation is complex and inefficient. Thus, deductive fault simulation method is a poor choice for circuits where some of the inputs may be unspecified. Such is the case for sequential circuits, where the values of some of the storage elements may remain unspecified for a long duration of a test sequence. Concurrent fault simulator [22] simulates a good circuit and all faulty circuits concurrently. How-

*Corresponding author. Phone: (608) 262-6490 Fax: (608) 262-1267 Email: saluja@engr.wisc.edu 
ever, only gates of a faulty circuit whose status (values on inputs and output of a gate) is different from that of the good circuit are explicitly simulated. Thus, it can perform 2-value as well as 3-value fault simulation equally efficiently. Concurrent fault simulation is often used as a reference fault simulator for performance comparison.

In recent literature some very efficient fault simulators have been reported. Three such simulators are 1) Differential fault simulator, 2) PROOFS,and 3) PARIS. Differential fault simulation [12] is a variation of serial fault simulation technique. Like serial fault simulation, after simulating the good machine, it simulates faulty machines, one by one, for each time frame. When it finishes simulation for each time frame, it stores only the state differences between the previously simulated and the the current simulated machines. This increases the simulation efficiency in terms of storage and processing time. PROOFS [11] is an extension of parallel fault simulation method [21]. In each time frame it simulates a set of faults determined by the word size of the host computer. The techniques used by a Differential fault simulator are also incorporated to further improve the performance. Another efficient fault simulator HOPE [20], also based on parallel fault simulation, was reported in the literature. PARIS (PARallel Iterative Simulator) [16] is an extension of parallel pattern single fault propagation simulation (PPSFP) method [3]. A number of test patterns are grouped based on the word size of the computer and a good machine is simulated for all these patterns. Following this, each faulty machine, one at a time, is also simulated for the group of patterns. A detected fault can not be dropped until the group of patterns is simulated.

Even though concurrent fault simulator is often used as a reference fault simulator for measuring the performance of a new fault simulator, the performance of the concurrent fault simulator itself is heavily dependent on the implementation. In this paper we propose an efficient fault simulation method for synchronous sequential circuits, which is a hybrid of concurrent and deductive fault simulation techniques. The basic concept is to identify inefficiencies in a conventional concurrent fault simulator and remove them systematically. In a concurrent fault sim- ulator, fault lists are implemented using linear linked lists and traversal operation on these lists is an inefficient and costly operation. A concurrent fault simulator also tends to use much memory since it must simulate good and all faulty machines simultaneously. Even though the differential representation of the good machine and a faulty machine is a salient feature of a concurrent fault simulator, its memory usage is often very high compared to other fault simulators. Thus, in order to improve the performance of a concurrent fault simulator, methods should be developed to process the fault lists efficiently and reduce the memory usage to minimum possible. HySim successfully fulfills these two goals. HySim also uses efficient representation of primitives using BDD-like representation and simplifies the fault deletion of fault descriptors associated with detected and converged faults.

In section 2, critical operations which cause inefficiencies in concurrent fault simulation are identified. Methods to overcome these inefficiencies and new approaches to improve the performance of fault simulation are described in section 3. Methods of memory usage reduction to be investigated are described in section 4 . Section 5 contains the experimental results and the discussion. The conclusion follows in section 6 .

\section{CRITICAL OPERATIONS IN CONCURRENT FAULT SIMULATION}

The performance of a fault simulator is measured by elapsed CPU time and its memory usage. Thus, the research efforts in fault simulation have been directed towards reducing the CPU time and the memory usage. In this section, we identify several critical operations which are related to fault list processing and cause inefficiencies in a concurrent fault simulator.

\subsection{Fault Deletion of Detected Faults}

Fault dropping is the deletion of detected faults from the fault list. Even in diagnostic applications, where 
simulation is used to build a fault dictionary, detected faults can be deleted from the fault list $[5,9]$. Thus, for deletion of faults the fault descriptors associated with the detected faults should be deleted from the data structure of the fault simulator. Here, the fault descriptor is an element of fault list associated with each gate. It represents the faulty gate, and holds the faulty gate specific informations such as fault index, input and output values and a pointer to the next fault descriptor. By deleting fault descriptors of the detected faults, the number of fault descriptors that are to be simulated is reduced. When and how to delete fault descriptors of detected faults is critical to the performance of a concurrent fault simulator. This problem was also identified by Gai et. al. [15]

\subsection{Removal of Converged Fault Descriptors}

This process is a little more complex than fault dropping. A fault descriptor is said to be converged if its status (input values and output value) becomes identical to that of the corresponding good gate. Note that the gates of a faulty circuit whose status is identical to the corresponding gates in the good circuit need not be simulated by a concurrent fault simulator. On the other hand a converged fault descriptor should be deleted because its non deletion can lead to erroneous results as explained below through two different examples identifying two possible scenarios.

Let us consider the circuit in Figure 1. Suppose this is a subcircuit embedded in a larger circuit. With a pattern $\mathrm{T} 1$ in which $\mathrm{xdefg}=11000$, the fault $\mathrm{x} / 0$, which represents line $x$ stuck-at 0 , is excited and propagated to the input to the gate $p$, but it is not observed at $\mathrm{p}$. With a pattern $\mathrm{T} 2$, in which signals $\mathrm{x}$ and e change, the fault is converged, i.e., the good value is same as the stuck-at value. Notice that even though the logic event dies at $\mathrm{m}$, fault descriptors of $\mathrm{x} / 0$ must be deleted from all the gates marked in Figure $1(b)$ including gates $m$ and $n$. If this fault descriptor is not deleted, it may later get propagated to the primary output and the fault may falsely be declared as detected. Thus, all occurrences of fault descriptor must be removed when the fault descriptor at the fault site is converged. If the fault was propagated

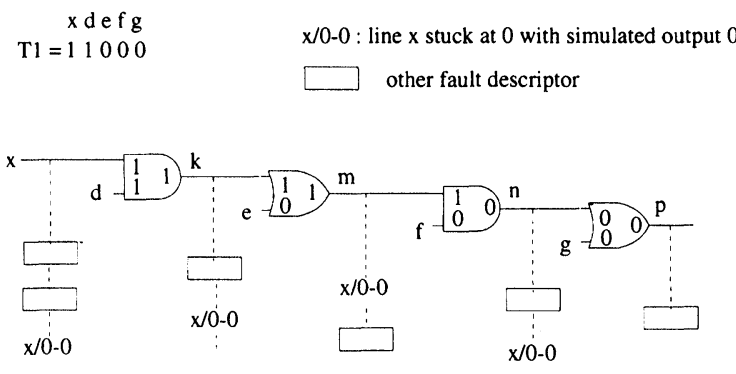

(a)
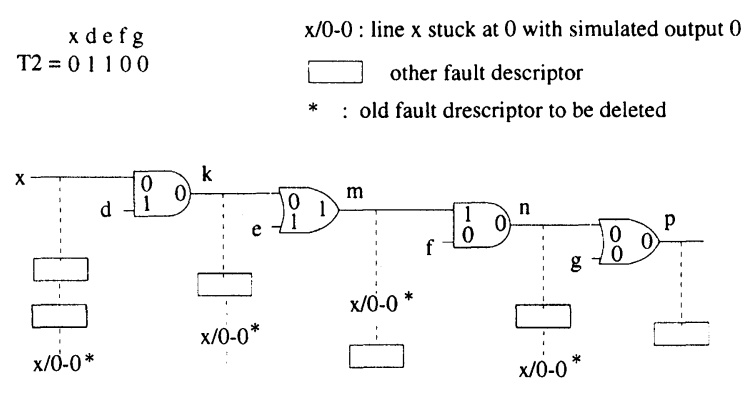

(b)

FIGURE 1 Example of Converged Fault Descriptor.

through multiple fanouts, the concurrent fault simulator must find all occurrences of a converged fault descriptor along the fanout branches. This selective deletion operation requires searching of the converged fault descriptor inside the fault lists associated with many gates in all possible paths from the converged fault site.

We now examine another situation which demonstrates that not all fault descriptors must be removed and in certain instances a more careful examination of the situation is required. Consider the situation shown in Figure 2. In this circuit the fault g/0 is propagated to a gate through more than one path. Sensitized paths are shown by bold lines. If the fault is converged at the fault site, all fault descriptors associated with the fault on the sensitized paths should be removed as in the first example. However, when a sensitized path is broken in the middle due to a controlling value on an input of a gate in a sensitized path, the fault propagated through multiple paths may or may not converge. In the circuit of Figure 2, a change in the value of $c$ does not cause convergence 

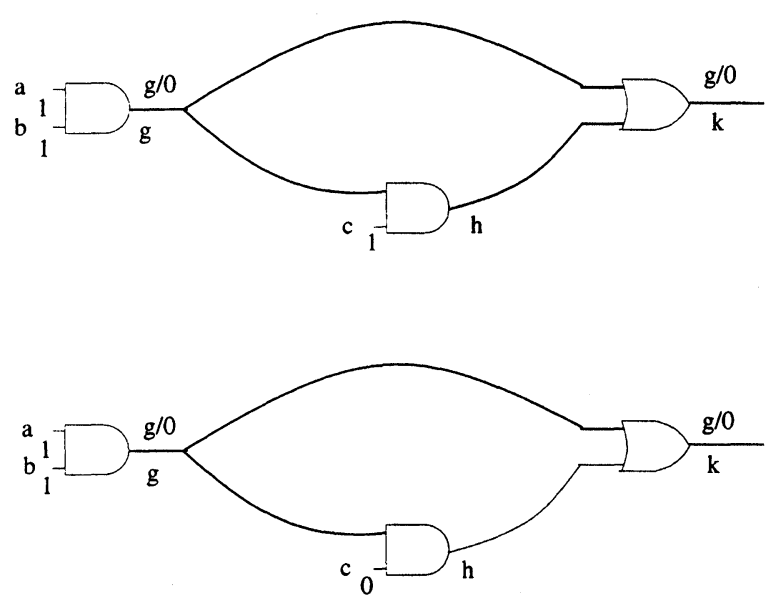

FIGURE 2 Selective Deletion of a Fault Descriptor.

of $\mathrm{g} / 0$ at gate $\mathrm{k}$, but will require deletion of $\mathrm{g} / 0$ from all gates on the path from gate $h$ to gate $k$. Thus, deletion operation must determine whether the fault descriptor propagated through multiple paths should be deleted or not. This can only be determined by the actual simulation of the fault descriptor. Our experience with most test sets indicates that such an occurrence is rare, nonetheless it should be handled correctly by the fault simulator in order to get the correct result.

\subsection{Memory Usage}

In concurrent fault simulation, only the gates of the faulty circuit whose status is different from the corresponding gates in the good circuit are explicitly simulated. Even though differential representation of faulty circuits in concurrent fault simulation appears very efficient, it cannot handle very large circuits because of limited memory and large number of faults in such circuits. Figure 3 (a) and (b), taken from [13], illustrate methods of representing faulty circuits using linear linked lists in concurrent and deductive fault simulators respectively. Concurrent fault simulator keeps a fault descriptor in the fault list associated with a gate whenever its status different from the good gate status. Thus, even if the output of the good and the faulty gate are identical the corresponding fault descriptor is entered in the list. In a deductive

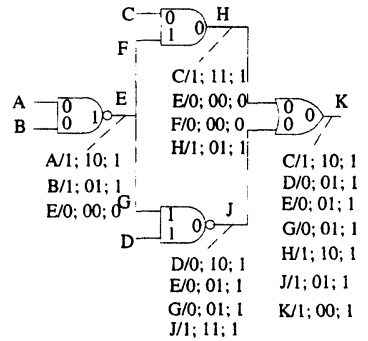

a) Concurrent fault simulation

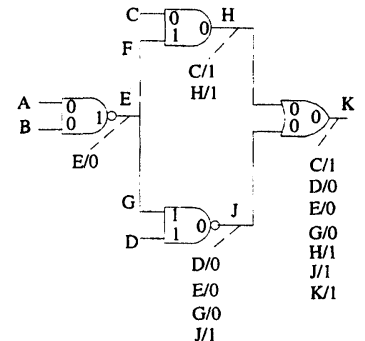

b) Deductive fault simulation
FIGURE 3 Fault Lists in a Concurrent and a Deductive Fault Simulators.

fault simulator, on the other hand, only the fault descriptor whose output value is different from the good is entered. We call a fault descriptor whose output value is identical to the output value of a corresponding gate in the good circuit an invisible fault descriptor (invisible fault in short) and fault descriptor whose output value is different from the output value off the corresponding gate of the good circuit a visible fault descriptor (visible fault in short). Thus, concurrent fault simulator has visible and invisible faults at each gate output while deductive fault simulator has only visible faults at each gate output. It follows that in this representation a deductive fault list is a subset of the concurrent fault list, and concurrent fault simulation requires more memory than deductive fault simulation. Furthermore, each fault descriptor in concurrent fault simulator requires more memory than deductive fault simulator because it has to store gate status information.

\subsection{List Traversal}

In a concurrent fault simulator, fault list for a gate is implemented as a linked list and is attached to the associated good gate. Individual fault descriptor in the fault list can be accessed by traversing the fault list. In order to increase the efficiency of the search process, the list is usually sorted in increasing (or decreasing) fault index order. This facilitates fault descriptor insertion due to fault divergence, fault descriptor deletion due to fault convergence, and fault 
descriptor evaluation. Detected fault identification also requires fault list traversal at primary outputs. Since the list traversal is an expensive operation, we must avoid redundant or unnecessary list traversals. Furthermore, since the list traversal time is directly proportional to the length of the list, it is beneficial to have shorter fault lists if possible. Typically a concurrent fault simulator maintains longer fault lists than deductive fault simulation, which in turn requires longer fault list traversal time.

\section{METHODS OF REMOVING INEFFICIENCIES}

In the previous section, we identified inefficiencies associated with critical operations in concurrent fault simulation. Below we describe a number of methods which can be used to improve the efficiency of a concurrent fault simulation for synchronous sequential circuits.

\subsection{Release-and-Reconstruct Method for Fault List Update}

Causes of inefficiency related to the removal of faults were identified in section 2.1 and 2.2. An efficient way of handling these problems is required to improve the performance of a concurrent fault simulator. We also indicated in the previous section that the problem of deletion of detected faults was noticed by Gai et. al. [15] as well. They experimented with various methods of fault dropping and proposed an asynchronous fault dropping method. Asynchronous fault dropping method deletes fault descriptors of a detected fault only when the associated fault lists are accessed and can be used for synchronous as well as asynchronous design. Their method marginally but demonstrably improved the performance of the simulator. However it suffers from two drawbacks. First, it requires more memory than necessary and second the performance is dependent on the test order which determines the activation of fault lists.

No efforts seem to have been made to remove the inefficiencies associated with deletion of converged fault descriptors. Our method concentrates on the deletion of converged fault descriptors. It reinitializes the data structure of the concurrent fault simulator before each test vector simulation.

Fault list must be updated according to the fault divergence and fault convergence whenever a new test vector is simulated. Updating can be done in two ways. The first method is to insert a new fault descriptor in the fault list whenever the associated fault diverges; and to delete an existing fault descriptor from the fault list whenever the associated fault converges. This method involves linear search operation in fault lists for the insertion and deletion of fault descriptors. This search operation is a rather inefficient operation. The second method is to delete the complete fault list associated with the gate before processing it and reconstruct the fault list. Reconstruction can be done mainly by append operation which is efficient. Thus, this method avoids expensive linear search operation and substitutes it by a list deletion and append operation. We call the first method a Modification method and the second method a Release-and-Reconstruct method. Release-andReconstruct method has been found to be more efficient than Modification method [18]. Further, Release-and-Reconstruct method can be implemented with very little overhead with multi-list traversal (MLT) [14]. MLT traverses the fault lists at inputs and output of a gate at the same time, thus, it can avoid unnecessary fault list traversals. All fault simulators developed for experiments and described in this paper use Release-and-Reconstruct method.

Time of deletion of fault lists that use Release-andReconstruct method is critical from the memory usage point of view because memory allocated to all fault descriptors associated with the deleted fault lists are returned to the memory pool for recycling. Memory management strategies for concurrent fault simulators described in section 5 of this paper are related to this aspect. 


\subsection{Rank Order Simulation}

Event driven method is a well established and commonly used method in simulation and fault simulation. However, the overhead of queue manipulation in event driven fault simulation often offsets the efficiency of it when the number of events becomes large. It is reported that an event driven simulation performs relatively poorly when the circuit activity becomes over $1 \%$ [23]. In event driven fault simulation for sequential circuits, an event is initiated by a primary input change, fault insertion, fault deletion due to fault divergence or convergence and state variable change. When multiple faults are to be processed simultaneously as in concurrent fault simulation, the sources of events increase tremendously since each fault can cause fault insertion, fault deletion and state change independently. Table I shows the percentage of list events in ISCAS 89 circuits during concurrent fault simulation. The activity is obtained by the formula

$$
\text { activity }=\frac{\# \text { fault lists processed }}{\# \text { vectors } \times \# \text { gates }} \times 100
$$

This table gives a measure of high activity in concurrent fault simulation. Thus, rank order simulation, where the circuit is levelized and the gates are processed in the level order, is a suitable choice for the concurrent fault simulation if the combined activity of the good and faulty circuits becomes high.

TABLE I List Events in Event Driven Concurrent Fault Simulator

\begin{tabular}{lrrrr}
\hline circuits & \# fault lists processed & \# vectors & \# gates & activity \\
\hline s298 & 21376 & 162 & 143 & $92 \%$ \\
s349 & 17140 & 91 & 197 & $95 \%$ \\
s526 & 160585 & 754 & 224 & $95 \%$ \\
s713 & 33658 & 107 & 471 & $67 \%$ \\
s832 & 111415 & 377 & 330 & $89 \%$ \\
s1238 & 124303 & 349 & 555 & $64 \%$ \\
s5378 & 945116 & 408 & 3043 & $76 \%$ \\
s35932 & 1484134 & 86 & 18149 & $95 \%$ \\
\hline
\end{tabular}

\subsection{Delayed Fault Descriptor Construction}

Fault descriptor can be constructed at the time when the first input difference is identified. This method requires updating of the fault descriptor if difference for other input occurs later, thus requiring unnecessary list traversals. Figure 4 shows an example which requires more than one list traversal. In Figure 4 (i) the fault list at the input a is processed, and fault descriptor $\mathrm{x}$ is propagated to the output of the gate and a new fault descriptor $\mathrm{x}$ is formed in the fault list at the output $c$. In Figure 4 (ii) the fault descriptor at the input $b$ is also to be propagated to the output of the gate, the fault list at the output is traversed again to access the fault descriptor $\mathrm{x}$ and update its status. If we delay fault descriptor construction at output until all input fault lists are processed as shown in Figure 5, we can avoid unnecessary list traversals. This can be achieved using MLT [14] by traversing all fault lists at inputs and output at the same time and construct a fault list at the output of a gate and evaluate each fault descriptor when it is constructed. Thus, one MLT is sufficient for construction and evaluation off a fault list at each gate output as opposed to two separate MLTs, i.e., evaluation MLT and propagation MLT [14]. When combined with rank order simulation, this method does not need fanout information for fault effect propagation, which in turn simplifies the data structure.

\subsection{Gate Value Evaluation Using BDD-Like Structure}

During fault simulation, the gates can be evaluated in various ways. Table lookup method is a popular

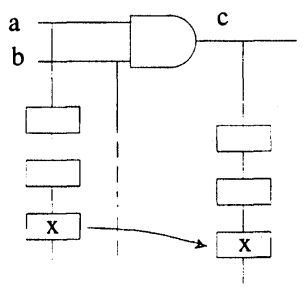

(i)

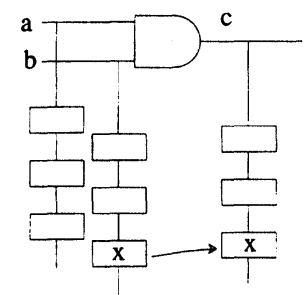

(ii)
FIGURE 4 Non-Delayed Fault Descriptor Construction. 


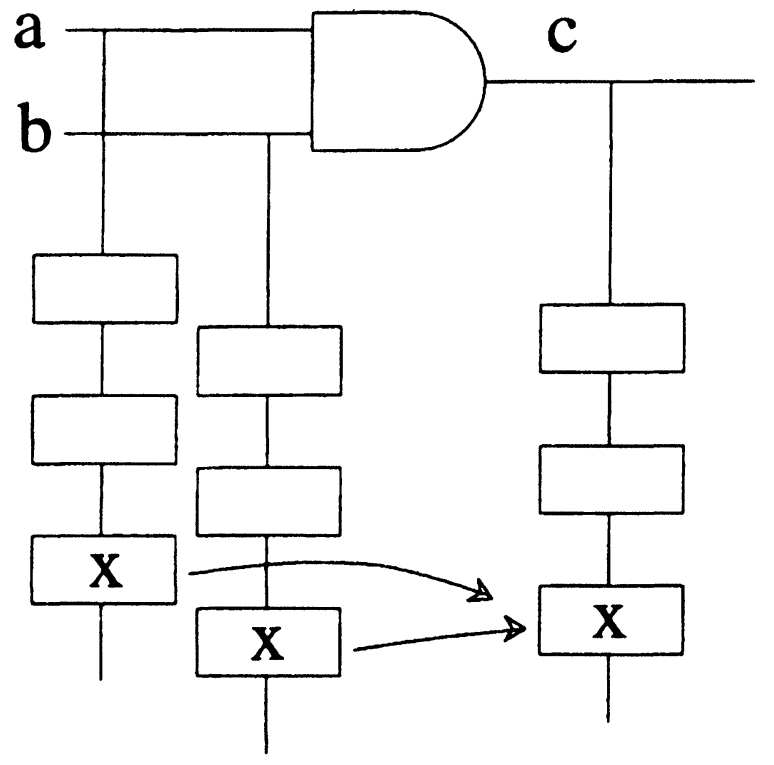

FIGURE 5 Delayed Fault Descriptor Construction.

method in use. Table lookup method requires packing operation and separate tables for different gate types. Further, the number of inputs that can be handled is restricted by the table size, and for the gates which have more inputs, multiple table lookups may be necessary. Another method is an iterative bit operation using bitwise AND, OR, and NOT instructions. It is the most efficient method when bit-level parallelism is utilized as in PPSFP/PARIS or parallel fault simulation/PROOFS.

BDDs (Binary Decision Diagram) are known to be very efficient for circuit simulation $[2,8]$. We can use BDD-like structure for gate value evaluation in concurrent fault simulation. Figure 6 shows the BDD for an $n$-input gate with controlling value $c$ and inversion parity i. If an input has a controlling value $\mathrm{c}$ left branch is taken, otherwise right branch is taken. At each node a flag is set if the input has $\mathrm{X}$ (unknown) value. At controlling leaf $C$, the gate value is $c \oplus i$. For AND, OR gates $i$ is 0 and for NAND, NOR and NOT gate $i$ is 1 . At the non-controlling leaf NC, we must check the flag if any of the input traversed was $X$. If so, the gate value becomes $X$, otherwise it becomes $\overline{\mathrm{c}} \oplus \mathrm{i}$. The experimental results given in section 5 justify the use off this method in our simulator.

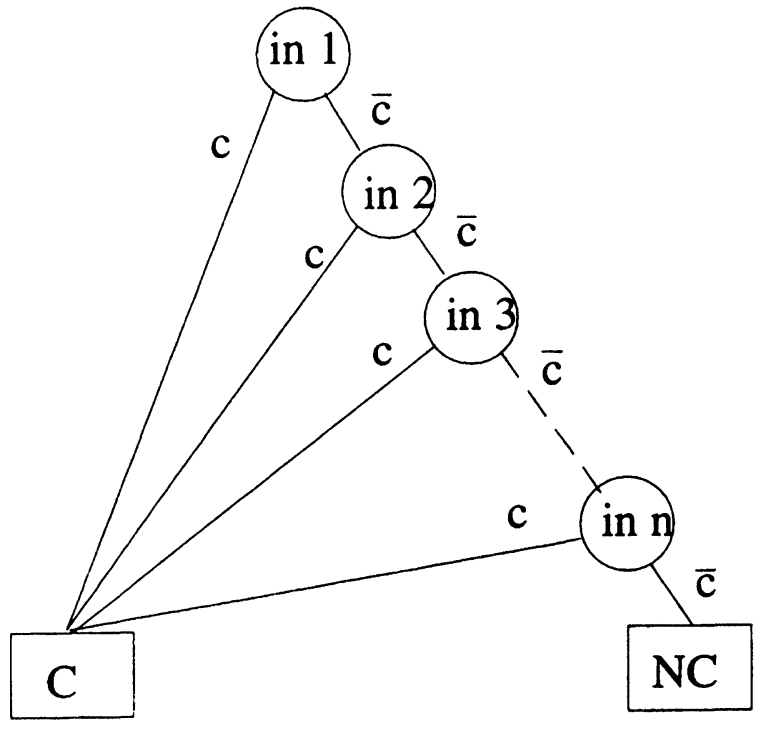

FIGURE 6 BDD-Like Evaluation of n-input gate.

\subsection{Visible Fault Only Fault List}

Concurrent fault simulation explicitly simulates a faulty gate whenever its input status (input values) and/or output status (output value) are different from good gate. Thus, typical concurrent fault simulators keep the fault descriptor in the fault list whenever its input and/or output values are different from good gate irrespective of its visibility. Note that invisible faults never propagate forward. Thus, deletion of invisible faults does not affect the correctness of the fault simulation. By deleting such faults from each fault list, we can shorten the fault list size, and thus reduce list traversal time and memory requirement. Better yet is not to construct the fault descriptors for the invisible faults at all, since visibility of the fault descriptors can be determined before construction. Notice that this is similar to deductive simulation which maintains only the visible faults in its fault list at each gate. However, the method is different from deductive fault simulation in the sense that fault list processing does not rely on set operations. All faulty machines and good machine are processed concurrently as in concurrent fault simulation even though fault lists look like that of deductive fault simulation. 


\section{METHODS FOR MEMORY USAGE REDUCTION}

Concurrent fault simulator is known to use more dynamic memory than other fault simulators. Usual techniques to cope with memory usage problem of concurrent fault simulation is to use memory pool for internal memory allocation for fault descriptors and fault set partitioning to reduce the number of faults that can be handled in each run. In this section, we will present the methods for reduction of dynamic memory usage. The methods are classified into two categories, i.e., memory management strategies and data representation strategies.

\subsection{Memory Management Strategies}

In this section, we investigate three memory management methods for Release-and-Reconstruction of fault lists. These are i) Lazy Method ii) Synchronous Method and iii) Eager Method. We explain these methods by introducing the concept of population map. As explained earlier, simulator essentially deletes, modifies and/or creates fault lists for different gates in the circuits. The population map is a pictorial representation of the circuit state during simulation. In the population map representation the circuit is divided into shaded and white areas. Gates in the shaded area of the population map may have associated fault lists, and gates in the white area have no fault lists associated with them. Note that white areas can occur because fault lists associated with gates in this area may have been released.

\subsubsection{Lazy Method}

Lazy method releases a fault list whenever the fault list is activated for update, and then constructs a new fault list. This process is repeated for each fault list to be updated. Lazy method does not release fault lists unless it has to, i.e., it tries to hold fault lists as long as it can. In this strategy, fault lists have a maximum life span. Conventional concurrent fault simulators use minor variations of this strategy. Figure 7 shows the population maps during the fault simulation that employs the Lazy method. Two sequences of maps shown in the figure correspond to the population maps for the consecutive test vectors. For the first test vector, the simulation starts with all white area in the population map since there is no fault lists built in the beginning. As the fault simulation proceeds, fault lists are created. Thus, shaded area grows and fills up the population map as shown in the three population maps shown in Figure 7 (a). For the second test vector, nearly all the population maps are shaded throughout the fault simulation because the fault lists built for the first test vector are retained by this method. This is shown in Figure 7 (b). The small white box in each picture represents a gate for which the associated fault list is deleted and reconstructed after the evaluation of the gate. As the fault simulation continues, the white box moves through all the gates in the first level and then all gates in the second level, and so on until all gates and their associated fault lists are processed. The population maps for the third and the subsequent test vectors change in the pattern similar to that for the second test vector.

\subsubsection{Synchronous Method}

Synchronous method was used to solve the problem of fault dropping and invalidated fault deletion [18]. In [18] this method is referred to as a Total Synchronism method and it was shown that this method not

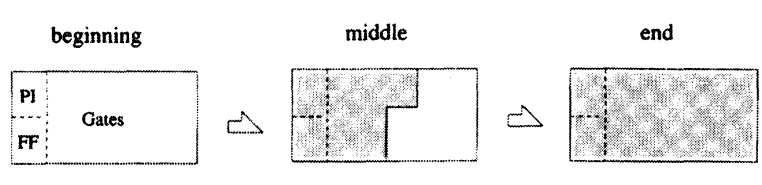

(a) maps for the first test vector

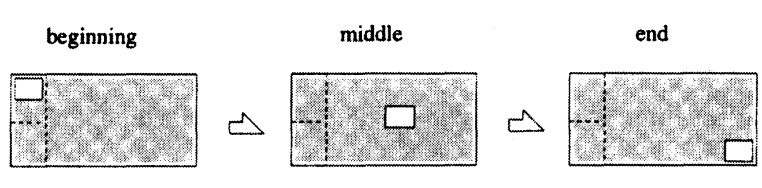

(b) maps for the second test vector

FIGURE 7 Progression of Population Maps for the Lazy Method. 
only handles the fault deletion problem "nicely", but also reduced the dynamic memory usage to a small degree. Gai et. al. [15] also noticed the fault deletion problem associated with fault dropping, but they did not focus on the memory usage issue. Synchronous method holds fault lists until fault lists associated with all gates and flip-flops are processed for a test vector. After the fault simulation is completed for a test vector, fault lists at all flip-flops are updated first, and then all fault lists except those at flip-flops are deleted. The deletion of fault lists can be done very efficiently. The lifespan of fault lists in Synchronous method is shorter than the Lazy method. Figure 8 shows the population maps during the fault simulation that employs the Synchronous method for the two consecutive test vectors. As before, the simulation starts with all white area in the population map and proceeds to construct fault lists for each gate as shown in Figure 8 (a). Thus, the population map is all white in the beginning, partially filled with shaded area in the middle and is all shaded in the end. For the second test vector, the simulation starts with a strip of shaded area in the beginning. This shaded area represents the flip-flops in the circuit, for which the associated fault lists are updated. As simulation proceeds, the shaded area grows and fills up the population map just like for the first test vector. This is shown in Figure 8 (b). For the third and the subsequent test vectors, the population map changes like that for the second test vector.

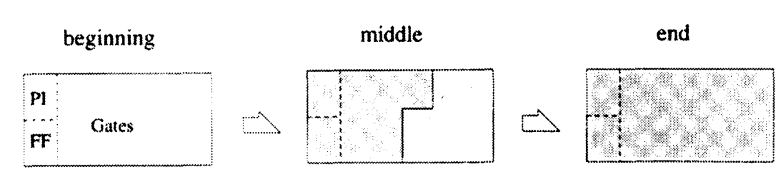

(a) maps for the first test vector

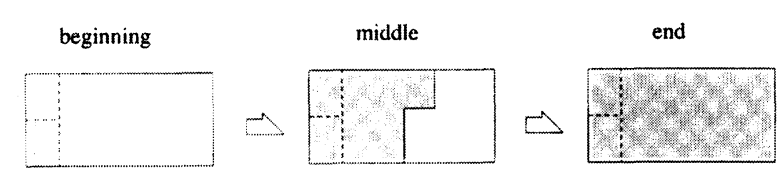

(b) maps for the second test vector

FIGURE 8 Progression of Population Maps for the Synchronous Method.

\subsubsection{Eager Method}

This method releases fault lists as soon as it is determined that the fault list is no longer needed. Each fault list maintains a reference counter. Whenever the fault list is accessed, the reference counter is incremented. If the number in the reference counter is same as the number of fanouts of the associated gate or flip-flop, the fault list is released immediately. Thus, in this method fault lists exist only as long as they are useful for simulation. Therefore, the fault lists in this method have the shortest life span among the three methods described. Figure 9 shows the population maps during fault simulation that employs the Eager method for two consecutive test vectors. For the first test vector, the fault simulation starts with all white area like the other two methods. However, as the simulation proceeds, it releases the fault lists as soon as they are found to be eligible for release. Thus, shaded area is generated as the new fault lists are created and the white area is generated as the used fault lists are released. So, the white area on the left side of the shaded area represents the gates whose fault lists are constructed and released. And the white area on the right side of the shaded area represents the gates that are not processed yet. In the end, fault lists are only at the gates that feed flip-flops. This progression is shown in Figure 9 (a). For the second test vector, the fault simulation starts with the fault lists at the gates that feed the flip-flops as shown in the picture marked "beginning" in Figure 9 (b). As

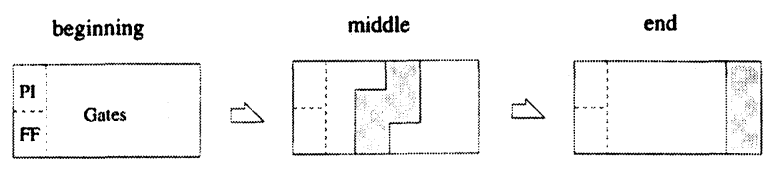

(a) maps for the first test vector

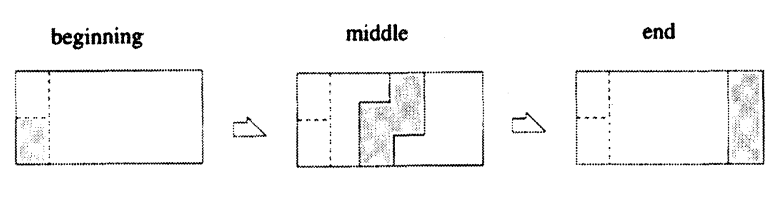

(b) maps for the second test vector

FIGURE 9 Progression of Population Maps for the Eager Method. 
the fault simulation proceeds, all fault lists in the shaded area are processed and then released. This in turn causes shaded area as shown in the picture marked "middle" in Figure 9 (b). As explained earlier, white area on the left of shaded area implies that all gates in that part have been processed whereas the gates in the white area on the right of shaded area remain to be processed. Finally the shaded area moves to the right as shown in the picture marked "end" in Figure 9 (b). The above process repeats for the third and the subsequent test vectors.

Clearly the fault lists in the Eager method have the shortest lifespan among the three methods. Also the size of the shaded area in the population map does not grow bigger than the other two methods throughout all three stages for each test vectors. On the other hand, the population map for the Lazy method is always filled up with shaded area for the second and the subsequent test vectors. And the shaded area in the population map of the Synchronous method grows and fills up the population map for every test vector. This implies that the Eager method holds smallest fault lists at any moment. Based on these arguments, we expect the Eager method to achieve the lowest memory usage.

\subsection{Data Representation Strategies}

In the previous section we proposed three memory management strategies. These strategies deal with the release of old fault lists. In this section we describe an efficient method of representing fault descriptors and a method to minimize the information in each fault list. Together these methods help reduce the dynamic memory usage in concurrent fault simulation for synchronous sequential circuits even further.

\subsubsection{Simplified Fault Descriptor Representation}

While forming the fault descriptors in the fault list associated with each gate, input values can be packed into a word (Input Status Word) and stored in each fault descriptor [19]. However, the packing of input values into status is a computationally expensive operation. Further, this is a redundant information since all these values can be obtained from the fault lists associated with the driving gates. If we do not use table lookup method for gate evaluation, we need not store input values for each fault descriptor in the fault list. Thus, by not storing input values in fault descriptors, we can reduce the size of individual fault descriptor in the fault list. Also, we can eliminate writing redundant information. Reduced fault descriptor size reduces the dynamic memory usage accordingly. Figure 10 shows the structures of a conventional and a simplified fault descriptor. A conventional fault descriptor must contain fault index, input status, output value and pointer fields in its structure whereas a simplified descriptor need only contain the fault index, the output value and the pointer fields. Assuming fault index, input status and pointer take 4 bytes and output value takes 1 byte, a conventional fault descriptor requires 13 bytes of memory while a simplified fault descriptor requires only 9 bytes of memory. Thus a simplified fault descriptor is $31 \%$ more efficient in memory usage. In a typical concurrent simulator a major part of memory is indeed associated with fault descriptors, hence simplified representation of fault descriptors is likely to reduce the memory requirement substantially.

\subsubsection{Visible Faults Only Fault List}

In section 3.4, Visible Faults Only Fault List method was proposed for performance improvement of a con-

non-simplified fault descriptor simplified fault descriptor

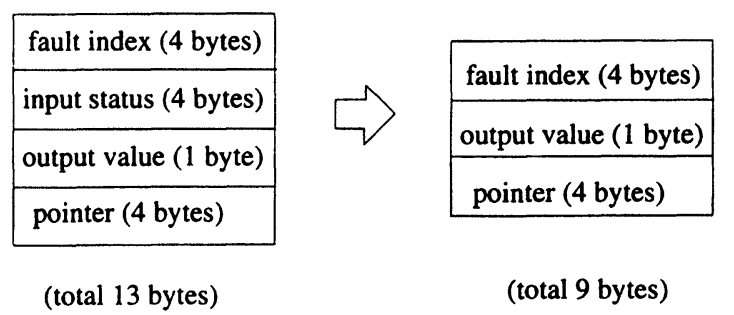

FIGURE 10 Byte Size of Fault Descriptors. 
current fault simulator. It was also mentioned that the length of the fault list is the same as that of deductive fault simulation. By not storing invisible faults in a fault list, the fault list length can be kept to its minimum and the memory usage is also reduced.

\section{IMPLEMENTATION OF THE PROPOSED METHODS AND EXPERIMENTAL RESULTS}

A series of different experiments were conducted step by step to determine the effectiveness of the methods proposed in the previous sections. First set of experiments was conducted to prove the effectiveness of Release-and-Reconstruct method used to solve the fault deletion problem mentioned in section 2 . The other proposed methods were verified in the successive experiments. All fault simulators for these experiments were written in the $\mathrm{C}$ language and were run on SPARCstation 2 under Unix environment.

\subsection{Verification of Release-and-Reconstruct Method}

We implemented three concurrent fault simulators which solve the fault deletion problem in three differ- ent ways to compare their relative performances. These are

1) FSim_1: Conventional concurrent fault simulator

2) FSim_2: Concurrent fault simulator with asynchronous fault dropping [15]

3) FSim_3: Concurrent fault simulator with Releaseand-Reconstruct method (Section 3.1)

For comparing the performance of the simulators the ISCAS combinational benchmark circuits [6] and a subset of ISCAS sequential benchmark circuits [7] were used. The characteristics of the circuits are summarized in Table II.

The performance of the concurrent fault simulators for ISCAS combinational and sequential benchmark circuits is summarized in Table III. The table also contains the number of test patterns in the each test set used for simulation and the corresponding fault coverages of these test sets. Relative performances of FSim_2 and FSim_3 with respect to FSim_1 are also given in the table. The relative performance of FSim_2 is included here to verify and prove the claims made by Gai et. al. [15]. It is evident that the Asynchronous Fault Deletion method implemented in FSim_2 improves the performance of a concurrent fault simulator (between $1 \%$ to $31 \%$ ), but such an improvement is only marginal compared to the im-

TABLE II Combinational and Sequential Circuits Tested

\begin{tabular}{|c|c|c|c|c|c|c|c|c|}
\hline \multicolumn{4}{|c|}{ Combinational Circuits } & \multicolumn{5}{|c|}{ Sequential Circuits } \\
\hline id & \# PI's & \# PO's & \# Gates & id & \# PI's & \#PO's & \# FF's & \# Gates \\
\hline $\mathrm{c} 432$ & 36 & 7 & 160 & s298 & 3 & 6 & 14 & 119 \\
\hline c499 & 41 & 32 & 202 & s349 & 9 & 11 & 15 & 161 \\
\hline c880 & 60 & 26 & 383 & s526 & 3 & 6 & 21 & 193 \\
\hline c1355 & 41 & 32 & 546 & s713 & 35 & 23 & 19 & 393 \\
\hline c 1908 & 33 & 25 & 880 & s832 & 18 & 19 & 5 & 287 \\
\hline c2670 & 233 & 140 & 1269 & s1238 & 14 & 14 & 18 & 508 \\
\hline c3540 & 50 & 22 & 1669 & s5378 & 35 & 49 & 179 & 2779 \\
\hline c5315 & 178 & 123 & 2307 & s35932 & 35 & 320 & 1728 & 16065 \\
\hline c6288 & 32 & 32 & 2416 & & & & & \\
\hline c7752 & 207 & 108 & 3513 & & & & & \\
\hline
\end{tabular}

id:ISCAS name of the benchmark circuit

\#PI's:Number of Primary Inputs

\#PO's:Number of Primary Outputs

\#FF's:Number of Flip-flops

\#Gates:Number of Total Gates in the circuit 
TABLE III Performance Comparison for ISCAS Circuits

\begin{tabular}{|c|c|c|c|c|c|c|c|}
\hline \multirow[b]{2}{*}{ circuits } & \multirow[b]{2}{*}{ \# vectors } & \multirow[b]{2}{*}{ coverage } & \multicolumn{3}{|c|}{ cpu time (sec) } & \multicolumn{2}{|c|}{ ratio } \\
\hline & & & FSim_1 & FSim_2 & FSim_3 & $\frac{\text { FSim_1 }}{\text { FSim_2 }}$ & $\frac{\text { FSim_1 }}{\text { FSim_3 }}$ \\
\hline c432 & 78 & $519 / 524$ & 1.27 & 1.18 & 0.68 & 1.08 & 1.87 \\
\hline c499 & 88 & $750 / 758$ & 5.28 & 4.85 & 2.47 & 1.09 & 2.14 \\
\hline c880 & 80 & $942 / 942$ & 2.00 & 1.85 & 1.20 & 1.08 & 1.67 \\
\hline c1355 & 126 & $1566 / 1574$ & 13.20 & 11.58 & 4.90 & 1.14 & 2.69 \\
\hline c1908 & 166 & $1869 / 1879$ & 10.90 & 10.80 & 6.20 & 1.01 & 1.76 \\
\hline c2670 & 249 & $2617 / 2747$ & 11.68 & 11.40 & 8.08 & 1.02 & 1.45 \\
\hline c3540 & 337 & $3295 / 3428$ & 36.00 & 32.10 & 21.63 & 1.12 & 1.66 \\
\hline c5315 & 218 & $5291 / 5350$ & 33.57 & 32.18 & 18.45 & 1.04 & 1.82 \\
\hline c6288 & 59 & $7710 / 7744$ & 179.10 & 136.38 & 93.78 & 1.31 & 1.91 \\
\hline c7752 & 381 & $7419 / 7550$ & 58.12 & 56.08 & 34.28 & 1.04 & $1: 67$ \\
\hline s298 & 162 & $264 / 308$ & 6.40 & 6.30 & 4.72 & 1.02 & 1.36 \\
\hline s349 & 91 & $335 / 350$ & 4.03 & 3.98 & 3.03 & 1.01 & 1.33 \\
\hline s526 & 754 & $418 / 555$ & 284.58 & 282.80 & 192.13 & 1.01 & 1.48 \\
\hline s713 & 107 & $470 / 581$ & 3.79 & 3.67 & 2.67 & 1.03 & 1.42 \\
\hline s832 & 377 & $708 / 870$ & 23.02 & 22.57 & 14.52 & 1.02 & 1.56 \\
\hline s1238 & 349 & $1283 / 1355$ & 6.97 & 6.75 & 5.80 & 1.03 & 1.20 \\
\hline s5378 & 408 & $3407 / 4603$ & 160.70 & 158.13 & 116.28 & 1.02 & 1.38 \\
\hline s35932 & 86 & $34398 / 39094$ & 1048.38 & 1039.82 & 519.90 & 1.01 & 2.02 \\
\hline
\end{tabular}

coverage: Detected Faults/Total Faults

FSim_1: Conventional Concurrent Fault Simulator

FSim_2: Concurrent Fault Simulator with Asynchronous Fault Dropping

FSim_3: Concurrent Fault Simulator with Release-and-Reconstruct Method

ratio: cpu time ratio

provement obtained by FSim_3. From Tables III it is evident that FSim_3 achieves $45 \%$ to $169 \%$ performance improvement over FSim_1 for combinational circuits and its improvement for sequential circuits is $20 \%$ to $102 \%$. FSim_3 improvement in performance over FSim_2 is between $16 \%$ and $136 \%$ for all the benchmark circuits. On the average FSim_3 is faster than FSim_1 and FSim $\_2$ by $69 \%$ and $59 \%$ respectively. This experiment proves that the Release-andReconstruct method implemented in FSim_3 is very effective for solving fault dropping and converged fault deletion problem.

\subsection{Verification of Rank Order Simulation and Delayed Fault Descriptor Construction}

A second set of experiments was conducted to determine the efficiency of the methods proposed in the section 3.2 and section 3.3. We built another fault simulator called FSim_4. Features included in the simulator are listed below.
FSim_4: Release_and_Reconstruct (Sec 3.1)

+ Rank Order Simulation (Sec 3.2)

+ Delayed Fault Descriptor Construction (Sec 3.3).

Table IV shows the elapsed CPU times of FSim_4 for selected ISCAS 89 sequential circuits. The performance of FSim_4 is consistently better than that of FSim_3 for all ISCAS sequential circuits tested.

TABLE IV Performance of FSim_4

\begin{tabular}{lrrr}
\hline circuits & \# vectors & coverage & $\begin{array}{c}\text { cpu time (sec) } \\
\text { FSim_4 }\end{array}$ \\
\hline s298 & 162 & $264 / 308$ & 1.80 \\
s349 & 91 & $335 / 350$ & 1.13 \\
s526 & 754 & $418 / 555$ & 40.05 \\
s713 & 107 & $470 / 581$ & 1.13 \\
s832 & 377 & $708 / 870$ & 7.03 \\
s1238 & 349 & $1283 / 1355$ & 3.65 \\
s5378 & 408 & $3407 / 4603$ & 53.22 \\
s35932 & 86 & $34398 / 39094$ & 165.57 \\
\hline
\end{tabular}


From the table, it is evident that the two methods incorporated in FSim_4 are very effective in improving the performance.

\subsection{Verification of BDD-Like Gate Evaluation and Memory Management Strategy Experiment}

Based on the results obtained in the section 5.1, we devised memory usage experiments as explained in the section 4.1 . The three memory management strategies, Lazy, Synchronous and Eager methods will be investigated in the following experiment. The experiment is to establish the best memory management strategy among the three strategies described in section 4.1. Note that the memory management strategy used in FSim_4 in section 5.2 is Synchronous method. We implemented three concurrent fault simulators. These are.

GSim_1: Concurrent fault simulator with Lazy Method (Section 4.1.1)

GSim_2: Concurrent fault simulator with Synchronous Method (Section 4.1.2)

GSim_3: Concurrent fault simulator with Eager Method (Section 4.1.3).

Since it is clear that Simplified Fault Descriptor takes less memory space than conventional fault descriptor, all fault simulators implemented for the experiments employ Simplified Fault Descriptor proposed in section 4.2.1. To verify the effectiveness of BDD-Like Gate Evaluation, we incorporated the method into GSim_1, GSim-2 and GSim_3. For comparing the memory usage of the simulators a subset of ISCAS sequential benchmark circuits [7] were used. The characteristics of the circuits are summarized in Table II.

Table V contains the dynamic memory usage of the simulators on the subset of ISCAS sequential circuits for test vectors obtained from STG-3 [10]. It showed that GSim_2 uses almost same amount of dynamic memory as GSim_1 in most cases. The improvement of GSim_3 over GSim_1 or GSim_2 is dramatic. The memory requirement of GSim_3 for the large circuits is as little as $14 \%$ of the memory requirement of GSim_1. Considering all the circuits memory requirement of GSim_3 was between $14 \%$ and $47 \%$ of the memory requirement of GSim_1. This proves the superiority of the Eager method over the Lazy and the Synchronous methods. We also obtained elapsed CPU times of GSim_1, GSim_2 and GSim_3, and included the measured CPU times in Table V. By comparing the performances of GSim's to that of the FSim's, it is obvious that BDD_Like Gate Evaluation achieved big performance improvement. However, it is observed that there is no significant performance differences between GSim_1, GSim_2 and GSim_3.

\subsection{Verification of Visible Fault Only Fault List Method}

We claimed in the previous sections 3.5 and 4.2.2 that Visible Faults Only Fault List would achieve both performance improvement and memory reduction. In order to prove this claim we built three fault simulators, called HySim_1, HySim_2 and HySim_3. Fea-

TABLE V Dynamic Memory Usage and Performance of FSim for Vectors from STG-3

\begin{tabular}{|c|c|c|c|c|c|c|c|c|}
\hline \multirow{2}{*}{ circuit } & \multirow{2}{*}{ \# vectors } & \multirow{2}{*}{ coverage (in \%) } & \multicolumn{3}{|c|}{ memory usage (in $\mathrm{Kb}$ ) } & \multicolumn{3}{|c|}{ cpu time (in sec) } \\
\hline & & & GSim_1 & GSim_2 & GSim_3 & GSim_1 & GSim_2 & GSim_3 \\
\hline s298 & 162 & 86 & 37.5 & 37.5 & 17.6 & 1.33 & 1.42 & 1.40 \\
\hline s349 & 91 & 96 & 53.9 & 53.9 & 12.9 & 0.77 & 0.82 & 0.75 \\
\hline $\mathrm{s} 526$ & 754 & 75 & 146.5 & 146.5 & 58.6 & 31.05 & 32.78 & 30.13 \\
\hline s713 & 107 & 81 & 92.6 & 85.6 & 17.6 & 0.77 & 0.85 & 0.72 \\
\hline s832 & 377 & 81 & 102.0 & 102.0 & 42.2 & 5.68 & 5.72 & 5.62 \\
\hline s1238 & 349 & 95 & 71.5 & 70.3 & 15.2 & 3.13 & 3.10 & 3.43 \\
\hline s5378 & 408 & 74 & 390.2 & 390.2 & 60.9 & 33.00 & 35.72 & 34.20 \\
\hline s35932 & 86 & 88 & 10787.1 & 10787.1 & 1531.6 & 128.30 & 129.97 & 131.62 \\
\hline
\end{tabular}


tures included in each of these simulators are listed below.

HySim_1:GSim_1 + Visible Fault Only Fault List HySim_2: GSim_2 + Visible Fault Only Fault List HySim_3: GSim_3 + Visible Fault Only Fault List

Visible Fault Only Fault List method evaluates a fault descriptor before creating it. If the calculated value is different from that of the corresponding good gate, a fault descriptor is actually created and appended to the fault list associated with the gate. Otherwise, the fault descriptor is not created at all. In other words, it stores only visible faults in a fault list like deductive fault simulation. Thus, we call the fault simulator HySim (Hybrid Fault Simulator). We tested the fault simulator for the same set of sequential circuits and test vectors. Table VI shows the memory usages of HySim's. Comparing Table V and Table VI, it is clear that the Visible Faults Only Fault List method achieved substantial improvement in memory usage reduction. For example, the memory requirements of $\mathrm{HySim}$ is $47 \%$ to $81 \%$ of the memory requirements of GSim_3. This implies that considerable number of fault descriptors in a fault list are invisible faults which need not be stored in a fault list.

We obtained test vectors generated by FASTEST [17] for a larger subset of ISCAS sequential circuits. We ran HySim_1, HySim_2 and HySim_3 for those test vectors. Table VII shows the characteristics of the circuits tested,the number of test vectors, fault coverage obtained and memory usages for the fault simulators. The results are consistent with the results for the test vectors from STG-3. That is, HySim_1 uses about same amount of dynamic memory as HySim_2. Whereas HySim_3 uses the least dynamic memory among the three fault simulators.

We measured elapsed CPU times of HySim_1, HySim-2 and HySim_3 to compare the performance. For comparison we obtained the results of PROOFS, a well-publicized state-of-the-art fault simulator. The Table VIII shows the results of HySim_1, HySim_2, HySim_3 and PROOFS for ISCAS sequential circuits for the test vectors generated by STG-3 [10]. Performance of HySim_1, HySim_2 and HySim_3 was found to be nearly identical for all the circuits used in our study. In all three cases the system memory was very large. We believe that for extremely large circuits that may require even larger memory space HySim_3 is likely to perform better than HySim_2 and HySim_3. However, HySim_1, HySim_2 and HySim_3 performed substantially better than GSim_1, GSim_2 and GSim_3 for all circuits tested, and all HySim's achieved better performance than PROOFS. We also ran HySim_3 and PROOFS for the test vectors obtained from FASTEST [17]. Table IX shows the ISCAS sequential circuits run for the test vectors from FASTEST and the results of these runs. We could not run PROOFS for s641, hence the entry is left blank for PROOFS in the Table IX. Note also that HySim_3 and PROOFS have different fault coverages for some circuits such as s208. It is because PROOFS enumerates and collapses faults internally while HySim does not. HySim accepts the fault list provided by a user. From the Table IX, it is evident that HySim_3 outperforms PROOFS for the test vectors obtained from FASTEST for all circuits tested. Further, from the performance data given in

TABLE VI Dynamic Memory Usage of HySim for Vectors from STG-3

\begin{tabular}{|c|c|c|c|c|c|}
\hline \multirow{2}{*}{ circuit } & \multirow{2}{*}{ \# vectors } & \multirow{2}{*}{ coverage (in \%) } & \multicolumn{3}{|c|}{ memory usage (in $\mathrm{Kb}$ ) } \\
\hline & & & HySim_1 & HySim_2 & HySim_3 \\
\hline s298 & 162 & 86 & 19.9 & 19.9 & 9.4 \\
\hline s349 & 91 & 96 & 38.7 & 38.7 & 9.4 \\
\hline s526 & 754 & 75 & 66.8 & 64.5 & 34.0 \\
\hline s713 & 107 & 81 & 66.8 & 58.6 & 11.7 \\
\hline s832 & 377 & 81 & 36.3 & 35.2 & 17.6 \\
\hline $\mathrm{s} 1238$ & 349 & 95 & 27.0 & 24.6 & 8.2 \\
\hline s5378 & 408 & 74 & 304.7 & 304.7 & 49.2 \\
\hline s35932 & 86 & 88 & 7655.9 & 7480.1 & 719.5 \\
\hline
\end{tabular}


TABLE VII Memory Usage of HySim for Vectors from FASTEST

\begin{tabular}{|c|c|c|c|c|c|c|c|c|c|}
\hline \multirow{2}{*}{ circuit } & \multirow{2}{*}{ \# PI's } & \multirow{2}{*}{ \# PO's } & \multirow{2}{*}{ \# FF's } & \multirow{2}{*}{ \# Gates } & \multirow{2}{*}{ \# vectors } & \multirow{2}{*}{ coverage } & \multicolumn{3}{|c|}{ Memory Usage (Kb) } \\
\hline & & & & & & & HySim_1 & HySim_2 & HySim_3 \\
\hline s208 & 11 & 2 & 8 & 96 & 4 & $18 / 217$ & 4.7 & 4.7 & 1.2 \\
\hline $\mathrm{s} 298$ & 3 & 6 & 14 & 119 & 132 & $259 / 308$ & 21.1 & 21.1 & 10.6 \\
\hline s344 & 9 & 11 & 15 & 160 & 88 & $329 / 342$ & 42.2 & 42.2 & 9.4 \\
\hline s349 & 9 & 11 & 15 & 161 & 84 & $335 / 350$ & 44.5 & 44.5 & 10.6 \\
\hline s382 & 3 & 6 & 21 & 158 & 50 & $196 / 399$ & 52.7 & 51.6 & 19.9 \\
\hline s386 & 7 & 7 & 6 & 159 & 121 & $287 / 384$ & 17.6 & 17.6 & 5.9 \\
\hline s400 & 4 & 6 & 21 & 164 & 56 & $250 / 424$ & 57.4 & 55.1 & 19.9 \\
\hline s420 & 19 & 2 & 16 & 196 & 7 & $28 / 455$ & 10.6 & 10.6 & 2.3 \\
\hline s444 & 3 & 6 & 21 & 181 & 59 & $265 / 474$ & 73.8 & 70.3 & 23.4 \\
\hline $\mathrm{s} 526$ & 3 & 6 & 21 & 173 & 15 & $63 / 555$ & 60.9 & 50.4 & 34.0 \\
\hline $\mathrm{s} 526 \mathrm{n}$ & 3 & 6 & 21 & 194 & 15 & $63 / 555$ & 64.5 & 53.9 & 34.0 \\
\hline s641 & 35 & 24 & 19 & 379 & 130 & $402 / 467$ & 22.3 & 21.1 & 3.5 \\
\hline s713 & 35 & 23 & 19 & 393 & 142 & $475 / 581$ & 27.0 & 25.8 & 4.7 \\
\hline $\mathrm{s} 820$ & 18 & 19 & 5 & 289 & 142 & $486 / 850$ & 34.0 & 32.8 & 17.6 \\
\hline $\mathrm{s} 832$ & 18 & 19 & 5 & 287 & 128 & $438 / 870$ & 29.3 & 28.1 & 15.2 \\
\hline $\mathrm{s} 838$ & 35 & 2 & 32 & 390 & 12 & $48 / 931$ & 29.3 & 28.1 & 4.7 \\
\hline s953 & 16 & 23 & 29 & 395 & 13 & $86 / 1079$ & 34.0 & 34.0 & 11.72 \\
\hline s1196 & 14 & 14 & 18 & 529 & 329 & $1239 / 1242$ & 38.7 & 38.7 & 8.2 \\
\hline $\mathrm{s} 1238$ & 14 & 14 & 18 & 508 & 345 & $1283 / 1355$ & 30.5 & 30.5 & 7.03 \\
\hline s1423 & 17 & 5 & 74 & 657 & 489 & $1293 / 1515$ & 221.5 & 221.5 & 43.4 \\
\hline s 1488 & 8 & 19 & 6 & 653 & 132 & $1093 / 1486$ & 126.6 & 126.6 & 28.1 \\
\hline s1494 & 8 & 19 & 6 & 647 & 141 & $1043 / 1506$ & 127.7 & 127.7 & 29.3 \\
\hline s5378 & 35 & 49 & 179 & 2779 & 904 & $3612 / 4603$ & 263.7 & 260.2 & 42.2 \\
\hline s9234 & 36 & 39 & 211 & 5597 & 52 & $470 / 6927$ & 1444.9 & 1441.4 & 213.3 \\
\hline
\end{tabular}

Table VIII and Table IX we can conclude that with the exception of three small circuits, HySim_3 runs between two to three times faster Than PROOFS.

HOPE [20] claimed to be more efficient than PROOFS though it was based on parallel fault simulation not on concurrent fault simulation. HOPE achieved similar speedup to HySim over PROOFS. We could not directly compare the performance of HySim with that of PARIS [16], for PARIS is not available to us for running on our machines under similar conditions.

\section{CONCLUSION}

We have identified critical operations which cause inefficiencies in conventional concurrent fault simulators and proposed several methods for performance improvement and dynamic memory usage reduction. A number of fault simulators based on concurrent fault simulation were implemented which incorporate one or more proposed methods. Through step-by-step experiment, we proved the effectiveness of the proposed methods. A fault simulator, HySim_3, which

TABLE VIII Performance of HySim's and PROOFS for Vectors from STG-3

\begin{tabular}{|c|c|c|c|c|c|c|}
\hline \multirow{2}{*}{ circuit } & \multirow{2}{*}{ \# vectors } & \multirow{2}{*}{ Coverage (in \%) } & \multicolumn{4}{|c|}{ cpu time (in sec) } \\
\hline & & & HySim_1 & HySim_2 & HySim_3 & PROOFS \\
\hline s298 & 162 & 86 & 1.13 & 1.13 & 1.13 & 0.88 \\
\hline s349 & 91 & 96 & 0.75 & 0.77 & 0.77 & 0.55 \\
\hline s526 & 754 & 75 & 21.05 & 21.32 & 21.08 & 13.55 \\
\hline s713 & 107 & 81 & 0.68 & 0.62 & 0.72 & 0.98 \\
\hline s832 & 377 & 81 & 4.60 & 4.33 & 4.35 & 7.57 \\
\hline $\mathrm{s} 1238$ & 349 & 95 & 2.40 & 2.28 & 2.45 & 4.42 \\
\hline s5378 & 408 & 74 & 27.82 & 28.55 & 28.53 & 52.18 \\
\hline s35932 & 86 & 88 & 101.98 & 101.02 & 101.28 & 179.32 \\
\hline
\end{tabular}


TABLE IX Performance of HySim_3 and PROOFS for Deterministic Test Vectors

\begin{tabular}{|c|c|c|c|c|c|c|c|c|c|}
\hline \multirow{2}{*}{ circuit } & \multirow{2}{*}{ \# PI's } & \multirow{2}{*}{ \# PO's } & \multirow{2}{*}{ \# FF's } & \multirow{2}{*}{ \# Gates } & \multirow{2}{*}{$\#$ vectors } & \multicolumn{2}{|c|}{ PROOFS } & \multicolumn{2}{|c|}{ HySim_3 } \\
\hline & & & & & & coverage & time & coverage & time \\
\hline s208 & 11 & 2 & 8 & 96 & 4 & $14 / 215$ & 0.05 & $18 / 217$ & 0.00 \\
\hline s298 & 3 & 6 & 14 & 119 & 132 & $259 / 308$ & 1.13 & $259 / 308$ & 0.73 \\
\hline s344 & 9 & 11 & 15 & 160 & 88 & $329 / 342$ & 0.82 & $329 / 342$ & 0.63 \\
\hline s349 & 9 & 11 & 15 & 161 & 84 & $335 / 350$ & 0.88 & $335 / 350$ & 0.63 \\
\hline s382 & 3 & 6 & 21 & 158 & 50 & $196 / 399$ & 1.65 & $196 / 399$ & 1.33 \\
\hline s386 & 7 & 7 & 6 & 159 & 121 & $280 / 382$ & 0.92 & $287 / 384$ & 0.32 \\
\hline$s 400$ & 4 & 6 & 21 & 164 & 56 & $250 / 424$ & 1.78 & $250 / 424$ & 1.47 \\
\hline$s 420$ & 19 & 2 & 16 & 196 & 7 & $24 / 430$ & 0.27 & $28 / 455$ & 0.03 \\
\hline s444 & 3 & 6 & 21 & 181 & 59 & $265 / 474$ & 2.30 & $265 / 474$ & 1.82 \\
\hline s526 & 3 & 6 & 21 & 173 & 15 & $63 / 555$ & 0.73 & $63 / 555$ & 0.45 \\
\hline s526n & 3 & 6 & 21 & 194 & 15 & $63 / 555$ & 0.73 & $63 / 555$ & 0.43 \\
\hline s641 & 35 & 24 & 19 & 379 & 130 & & & $402 / 467$ & 0.62 \\
\hline s713 & 35 & 23 & 19 & 393 & 142 & $475 / 581$ & 1.22 & $475 / 581$ & 0.83 \\
\hline s820 & 18 & 19 & 5 & 289 & 142 & $486 / 850$ & 5.67 & $486 / 850$ & 1.42 \\
\hline s832 & 18 & 19 & 5 & 287 & 128 & $438 / 870$ & 5.53 & $438 / 870$ & 1.42 \\
\hline s838 & 35 & 2 & 32 & 390 & 12 & $42 / 857$ & 1.03 & $48 / 931$ & 0.17 \\
\hline s953 & 16 & 23 & 29 & 395 & 13 & $86 / 1079$ & 1.83 & $86 / 1079$ & 0.27 \\
\hline s1196 & 14 & 14 & 18 & 529 & 329 & $1239 / 1242$ & 6.22 & $1239 / 1242$ & 2.08 \\
\hline s1238 & 14 & 14 & 18 & 508 & 345 & $1283 / 1355$ & 10.22 & $1283 / 1355$ & 2.28 \\
\hline s1423 & 17 & 5 & 74 & 657 & 489 & $1293 / 1515$ & 37.6 & $1293 / 1515$ & 21.82 \\
\hline s1488 & 8 & 19 & 6 & 653 & 132 & $1011 / 1417$ & 11.62 & $1093 / 1486$ & 3.38 \\
\hline s1494 & 8 & 19 & 6 & 647 & 141 & $1147 / 1459$ & 11.78 & $1043 / 1506$ & 3.45 \\
\hline s5378 & 35 & 49 & 179 & 2779 & 904 & $3612 / 4603$ & 175.32 & $3612 / 4603$ & 57.05 \\
\hline s9234 & 36 & 39 & 211 & 5597 & 52 & $470 / 3938$ & 52.20 & $470 / 6927$ & 31.62 \\
\hline
\end{tabular}

Fault coverage for s208, s386, s420, s838, s1488, s1494 and s9234 are different for PROOFS and HySim_3. (See text)

incorporates all the proposed methods achieved substantial improvement in both performance and dynamic memory usage. Indeed, our experimental results show that HySim_3 is very efficient and outperforms all the known and available fault simulators.

\section{Acknowledgements}

Research work reported here is supported in part by the National Science Foundation grant MIP-9111886 and the AT\&T Foundation.

\section{References}

[1] M. Abramovici, M. A. Breuer, and A. D. Friedman, Digital Systems: Testing and Testable Design. New York: Computer Science Press, 1990.

[2] S. B. Akers, "Binary Decision Diagram," IEEE Trans. on Computers, vol. C-27, pp. 509-516, June 1978.
[3] J. A. Waicukauski et al, "Diagnosis of BIST Failures by PPSFP Simulation," Proc. IEEE Intl. Test Conference, pp. 480-484, September 1987.

[4] D. B. Armstrong, "A Deductive Method for Simulating Faults in Logic Circuits," IEEE Trans. Comput., vol. C-21, pp. 464-471, 1972.

[5] M. A. Breuer and A. D. Friedman, Diagnosis and Reliable Design of Digital Systems. Computer Science Press, 1976.

[6] F. Brglez and H. Fujiwara, "A Neutral Netlist of 10 Combinational Benchmark Circuits," Proc. Intl. Symp. on Circuits and Systems, pp. 705-712, 1985.

[7] F. Brglez, D. Bryan, and K. Kozminski, "Combinational Profiles of Sequential Benchmark Circuits," Proc. Intl. Symp. on Circuits and Systems, pp. 1929-1934, 1989.

[8] R. E. Bryant, "Graph-Based Algorithms for Boolean Function Manipulation," IEEE Trans. on Computers, vol. C-35, pp. 677-691, August 1985.

[9] P. Camurati, A. Lioy, P. Prinetto, and M. S. Reorda, "Diagnostic Oriented Test Pattern Generation," in Proc. EDAC90, Glasgow, U.K., pp. 470-474, March, 1990.

[10] W. Cheng and S. Davidson, "Sequential Circuit Test Generator (STG) Benchmark Results," International Symposium of Circuits \& Systems, pp. 1938-1941, May 1989.

[11] W. Cheng and J. H. Patel, "PROOFS: A Super Fast Fault Simulator for Sequential Circuits," Proc. Europ. Design Automation Conference, pp. 475-479, 1990.

[12] W. T. Cheng and M. L. Yu, "Differential Fault Simulation 
for Sequential Circuits," Journal of Electronic Testing: Theory and Applications, pp. 7-13, 1990.

[13] H. Fujiwara, Logic Testing and Design for Testability. Cambridge, MA: The MIT Press, 1985.

[14] S. Gai, P. L. Montessoro, F. Somenzi, and E. Ulrich, "MOZART, a Concurrent Multilevel Simulator," Trans. on Computer Aided Design, vol. 6, pp. 1006-1012, November 1987.

[15] S. Gai and P. L. Montessoro, "The Fault Dropping Problem in Concurrent Event-Driven Simulation," IEEE Trans. Computer-Aided Design, vol. 10, pp. 968-971, August, 1991.

[16] N. Gouders and R. Kaibel, "PARIS: A Parallel Pattern Fault Simulator for Synchronous Sequential Circuits," IEEE Int. Conference on Computer Aided Design, pp. 542-545, November 1991.

[17] T. P. Kelsey, K. K. Saluja, and S. Lee, "An Efficient Algorithm for Sequential Circuit Test Generation," IEEE Transactions on Computers, pp. 1361-1371, November 1993.

[18] K. Kim and K. K. Saluja, "On Fault Deletion Problem in Concurrent Fault Simulation for Synchronous Sequential Circuits," IEEE VLSI Test Symposium, April, 1992.

[19] D. H. Lee and S. M. Reddy, "On Efficient Concurrent Fault Simulation For Synchronous Sequential Circuits," Submitted for publication.

[20] H. K. Lee and D. S. Ha, "HOPE: An Efficient Parallel Fault Simulator for Synchronous Sequential Circuits," IEEE Design Automation Conference, pp. 336-340, June, 1992.

[21] S. Seshu, "On an Improved Diagnosis Program," IEEE Trans. Elect. Comput., vol. EC-12, pp. 76-79, 1965.

[22] E. G. Ulrich and T. Baker, "The Concurrent Simulation of
Nearly Identical Digital Networks," Proc. of 10th Design Automation Workshop, vol. 6, pp. 145-150, 1973.

[23] Z. Wang and P. M. Maurer, "LECSIM: A Levelized Event Driven Compiled Logic Simulator," IEEE Design Automation Conference, pp. 491-496, June 1990.

\section{Authors' Biographies}

Kyuchull Kim received M. S. and Ph. D. degrees in Electrical and Computer Engineering from the University of Wisconsin-Madison in 1986 and 1992 respectively. He is currently a faculty at Dankook University, Korea.

Kewal K. Saluja is a Professor in the Department of Electrical and Computer Engineering at the University of Wisconsin-Madison. His research interests include test generation, fault simulation, design for testability and built-in self-test. He has published over 100 refereed journal and conference papers in these areas, $\mathrm{He}$ is currently the Associate Editor for the letters sections of the Journal of Electronic Testing: Theory and Applications (JETTA). 

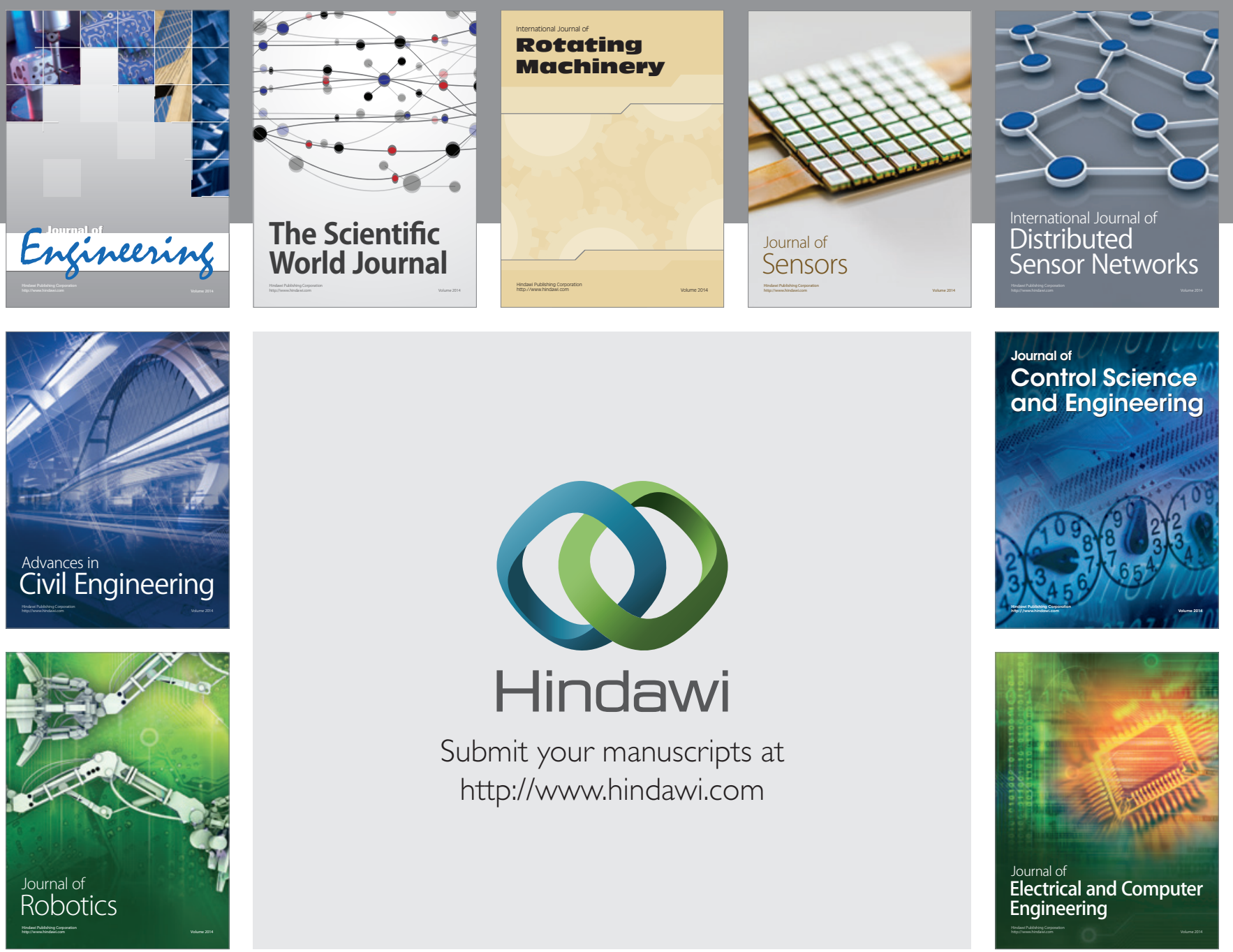

Submit your manuscripts at

http://www.hindawi.com
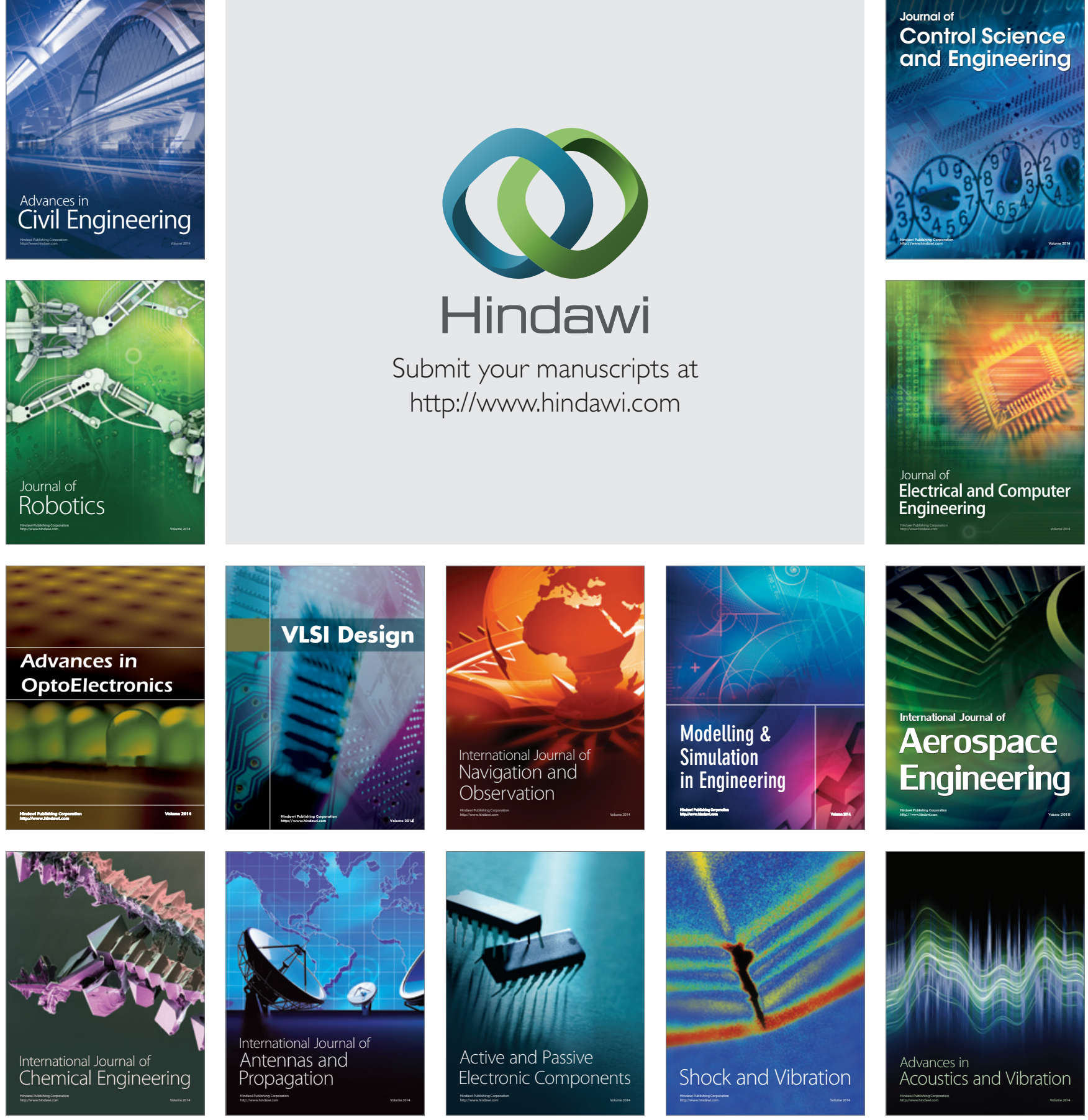